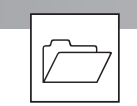

Endokrynologia Polska

DOI: $10.5603 /$ EP.a2021.0027

Volume/Tom 72; Number/Numer 3/2021 ISSN 0423-104X, e-ISSN 2299-8306

\title{
Giant silent corticotrope pituitary adenoma in a patient with complicated clinical course
}

\author{
Ognian Kalev' ${ }^{1}$ Marina Hodolic ${ }^{2}$,Berndt Tomancok ${ }^{3}$, Michael Sonnberger ${ }^{4}$, Markus Hutterer ${ }^{5}$, \\ Daniela Ehrlich ${ }^{6}$, Robert Pichler ${ }^{7}$
}

\author{
${ }^{1}$ Institute of Pathology and Neuropathology, Neuromed Campus, Kepler University Hospital, Linz, Austria \\ ${ }^{2}$ Nuclear Medicine Research Department, IASON, Graz, Austria; Nuclear Medicine Department, Faculty of Medicine and \\ Dentistry, Palacký University Olomouc, Olomouc, Czech Republic \\ ${ }^{3}$ Department of Neurosurgery, Neuromed Campus, Kepler University Hospital, Linz, Austria \\ ${ }^{4}$ Institute of Neuroradiology, Neuromed Campus, Kepler University Hospital, Linz, Austria \\ ${ }^{5}$ Department of Neurology, Neuromed Campus, Kepler University Hospital, Linz, Austria \\ ${ }^{6}$ Department of Gerontology, Neuromed Campus, Kepler University Hospital, Linz, Austria \\ ${ }^{7}$ Institute of Nuclear Medicine, Neuromed Campus, Kepler University Hospital, Linz, Austria
}

Key words: acromegaly; Cushing's disease; somatostatin receptor subtype; immunohistochemistry; somatostatin analogues

Most pituitary adenomas are benign and exhibit slow expansive growth; however, about a third of pituitary adenomas are invasive, and some of them exhibit more aggressive clinical behaviour [1]. Surgical and radiation treatment are the cornerstones of clinical management, further medical treatment options are efficient mostly for endocrine active tumours. We present a case of a pituitary adenoma with ACTH-positive immunochemistry without clinical or endocrine laboratory markers of Cushing's disease, and our considerations for the selection of therapeutic options.

A then 17-year-old male patient had his first pituitary operation in 2003, revealing a clinically silent immunohistochemically ACTH-positive pituitary adenoma (Fig. 1). He presented in our hospital in 2019 because of epileptic seizures for the first time; he had previously been living in Styria. Since his diagnosis 4 transsphenoidal and transcranial operations had been effectuated as well as radiation therapy (2007 and gamma knife in 2010) and an unsuccessful medical approach with temozolomide. Nonetheless, continuous tumour growth had taken place, causing visual restrictions because of compression of the chiasma opticum. Remarkably, his Ki-67 level had always been $<4 \%$. MRI presented a 7.5-cm tumour relapse in April 2019.

Endocrine evaluation showed central hypothyroidism and testosterone deficiency, which were substituted; the adrenocortical axis appeared normal as at any time point. Constant visual field impairment was present and some

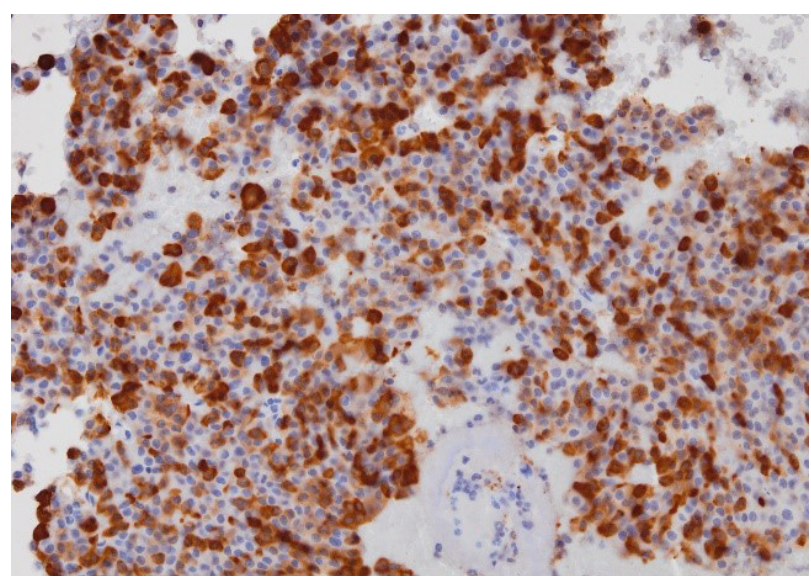

Figure 1. More than $10 \%$ of the tumour cells stained positive for ACTH, magnification $20 \times$

mood disturbances; otherwise, the patient was in good clinical conditions. The medical record included a history of polytoxic drug abuse. In search of a possible medical approach, we discussed the therapies used in Cushing's disease targeting the pituitary [2]. PET imaging was done with 18 F-Fallypride, which is specific for dopamine receptor type $2[3,4]$. The tumour showed marked uptake of the tracer (Fig. 2), and we indicated therapy with cabergoline at $2 \mathrm{mg}$ /week. We also measured SSTR immunohistochemistry on preserved tumour material which was negative for subtype 2 and 5 (Fig. 3 and 4), so a possible target for pasireotide was not present. Otherwise, PD-L1 was positive in $70 \%$ of all tumour cells. Therefore, specific 


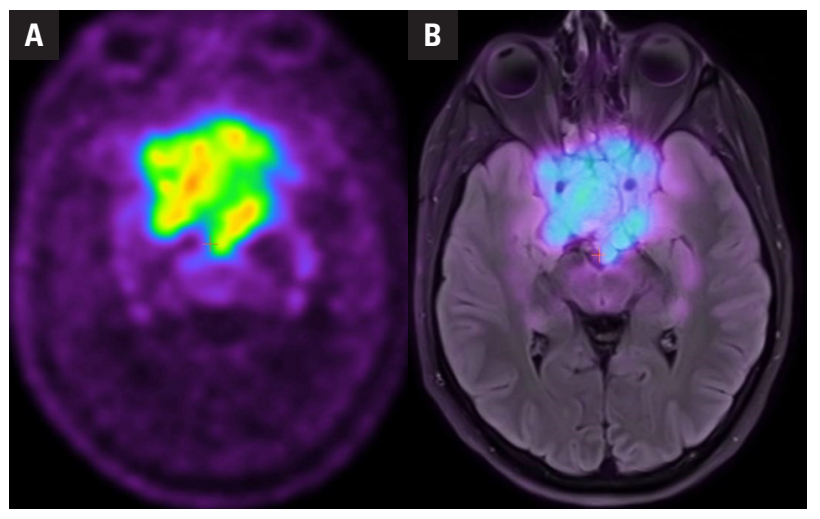

Figure 2A. ${ }^{18} \mathrm{~F}$-Fallypride PET presented high D2-receptor targeting uptake of the tumour with a SUVmax $=13$, comparable to the physiological high retention of the putamen; B. PET images were also fused to MRI

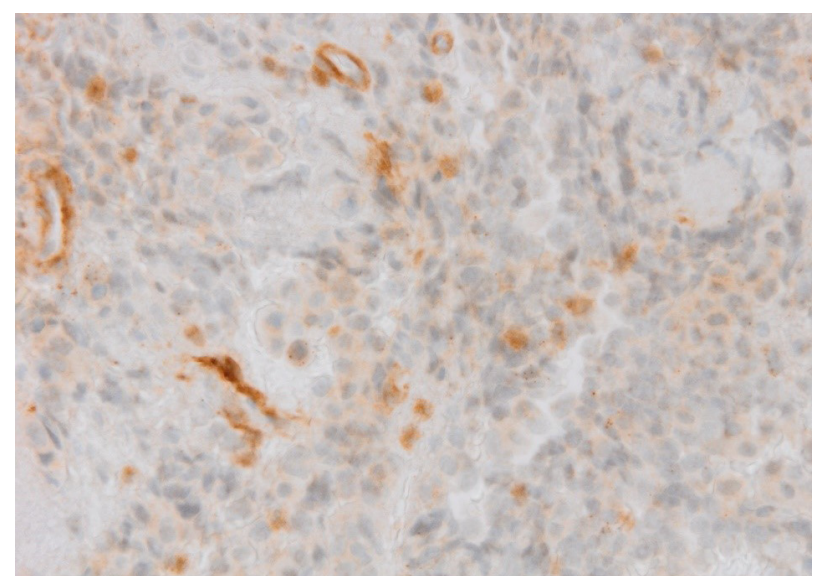

Figure 3. The tumour cells are SSTR2-negative, magnification $40 \times$

antibody therapy with nivolumab was started in June 2019. He received in total 9 cycles of nivolumab until reaching the limiting dose. Stable disease was observed by MRI in November 2019. Then the patient refused any further tumour-specific therapy; clinical worsening including diminishing vision and reappearance of seizures took place. MRI control in May 2020 showed a marked tumour progress, and so palliative care was organized.

Silent corticotrope adenomas are rare entities, giant adenomas, in particular, seldom occur. Aggressive behaviour would have been expected to be related to a high Ki-67 index, which was not the case here. This circumstance may also partially explain the disappointing results of radiation therapy.

The search for medical therapeutic options in this case is problematic because any medication in this therapeutically refractory constellation of a clinically silent ACTH-positive pituitary adenoma has to be considered experimental. Corticotrope adenomas with invasive growth have been shown to lose SSTR5 and D2 receptor expression [5]. Because there was no significant expres-

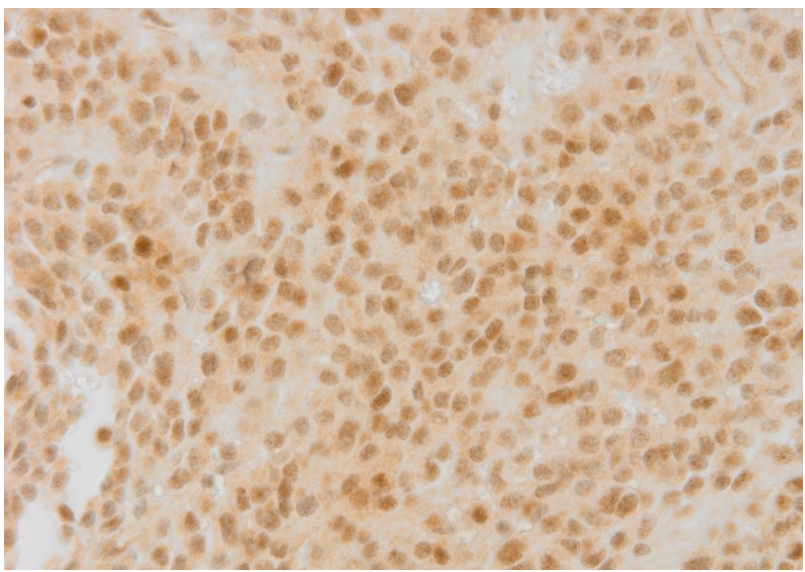

Figure 4. The weak, fine granular, mostly nuclear SSTR5-positivity is non-specific, the cell membranes appear SSTR5-negative, magnification $40 \times$

sion of SSTR subtype 2 or 5 the tumour board decided against a trial with pasireotide. Conservative therapy with dopamine agonists is effective in cases of clinically non-functioning pituitary adenomas, depending on the expression of D2 receptors [6]. This condition could be proven via D2 receptor $\mathrm{PET} / \mathrm{CT}$, and therefore a specific endocrine therapy was initiated. The decision for additional antibody therapy with nivolumab was based on the pathohistological findings. A role for immunohistochemistry in the evaluation of tumour specimen and receptor-based nuclear medicine imaging was present here for decision-making in personalized medicine.

\section{Funding}

This research received no external funding.

\section{Conflicts of interest}

The authors declare no conflict of interest.

\section{References}

1. Dai C, Liu X, Ma W, et al. The Treatment of Refractory Pituitary Adenomas. Front Endocrinol (Lausanne). 2019; 10: 334, doi: 10.3389/fendo.2019.00334, indexed in Pubmed: 31191457.

2. de Bruin C, Feelders RA, Lamberts SWJ, et al. Somatostatin and dopamine receptors as targets for medical treatment of Cushing's Syndrome. Rev Endocr Metab Disord. 2009; 10(2): 91-102, doi: 10.1007/s11154-008-9082-4, indexed in Pubmed: 18642088.

3. de Herder WW, Reijs AEM, Feelders RA, et al. Diagnostic imaging of dopamine receptors in pituitary adenomas. Eur J Endocrinol. 2007; 156 Suppl 1: S53-S56, doi: 10.1530/eje.1.02349, indexed in Pubmed: 17413189.

4. Liu H, Zakiniaeiz Y, Cosgrove KP, et al. Toward whole-brain dopamine movies: a critical review of PET imaging of dopamine transmission in the striatum and cortex. Brain Imaging Behav. 2019; 13(2): 314-322, doi: 10.1007/s11682-017-9779-7, indexed in Pubmed: 29071465.

5. de Bruin C, Pereira AM, Feelders RA, et al. Coexpression of dopamine and somatostatin receptor subtypes in corticotroph adenomas. J Clin Endocrinol Metab. 2009; 94(4): 1118-1124, doi: 10.1210/jc.2008-2101, indexed in Pubmed: 19141584.

6. Gabalec F, Beranek M, Netuka D, et al. Dopamine 2 receptor expression in various pathological types of clinically non-functioning pituitary adenomas. Pituitary. 2012; 15(2): 222-226, doi: 10.1007/s11102-011-0316-1, indexed in Pubmed: 21597974. 